\title{
Rainbow spanning subgraphs in bounded edge-colorings of graphs with large minimum degree
}

\author{
Pilar Cano* 1,2 , Guillem Perarnau $^{\dagger} 3$, and Oriol Serra ${ }^{\ddagger} 1$ \\ ${ }^{1}$ Departament de Matemàtiques Universitat Politècnica de Catalunya, Barcelona, Spain. \\ ${ }^{2}$ School of Computer Science, Carleton University, Ottawa, Canada. \\ ${ }^{3}$ School of Mathematics, University of Birmingham, Birmingham, United Kingdom.
}

March 2, 2017

\begin{abstract}
In this paper we study the existence of rainbow perfect matching and Hamiltonian cycles under minimum degree conditions on an edge-colored graph where every color appears a bounded number of times. We derive asymptotically tight bounds on the minimum degree for the existence of such rainbow spanning structures.
\end{abstract}

\section{Introduction}

An edge-coloring of $G$ is $r$-bounded if every color appears in at most $r$ edges of $G$. A subgraph $H$ of an edge-colored graph $G$ is rainbow if all the edges of $H$ have a distinct color.

Erdős and Spencer in [6] initiated the study of rainbow spanning structures motivated by a problem of Ryser on the existence of Latin transversals in Latin squares [11. They showed that every $k$-bounded edge-coloring of the complete bipartite graph $K_{n, n}$ admits a rainbow perfect matching, provided that $k \leq(n-1) /(4 e)$.

Albert, Frieze and Reed in [1] studied the existence of rainbow Hamiltonian cycles in $k$-bounded edge-colored complete graphs $K_{n}$, provided that $k \leq(n-1) / 64$. In a more general setting, Böttcher, Kohayakawa and Proccaci [3] proved that for every graph $H$ on $n$ vertices and maximum degree at most $\Delta$, there exists a rainbow copy of $H$ in any $k$-bounded coloring of $K_{n}$, provided that $k \leq n /\left(51 \Delta^{2}\right)$. Their approach used the framework developed by Lu and Székely [10] on negatively dependency graphs constructed from matchings. While the constant 51 is clearly not optimal, the dependence on $\Delta$ of the result in [3] is best possible. In fact, as a by-product of the use of the Lovász Local Lemma, all the upper bounds on $k$ are not tight.

Other related results include the existence of almost spanning rainbow trees in $K_{n}$ [7], the existence of spanning rainbow multipartite graphs with bounded degrees in multipartite complete graphs $K_{n, n \ldots, n}$ [8] as well as the existence of rainbow subhypergraphs in complete hypergraphs [ 5 , 8. All these results hold provided that the edge-coloring of the host graph is $k$-bounded for an appropriate value of $k$.

*Email: m.pilar.cano@upc.edu

${ }^{\dagger}$ Email: g. perarnau@bham.ac.uk.

${ }^{\ddagger}$ Email: oriol . serra@upc. edu. 
A natural question is whether one can still find a rainbow copy of a spanning subgraph $H$ in a host graph $G$ that is sparser than the corresponding complete graph structure (complete graphs, complete multipartite graphs, complete hypergraphs ...). Obviously, a necessary condition is that $G$ must contain at least one copy of $H$. Among other conditions, the existence of a spanning subgraph can be guaranteed by requiring $G$ to have a large minimum degree.

Sudakov and Volec gave a first step on this direction by proving the following (see the concluding remarks in [13], or Theorem 1.7 in [8] for a formal statement).

Theorem 1 ([13]). There exist positive constants $\beta$ and $c$ such that the following holds. Let $H$ be a graph on $n$ vertices of maximum degree $\Delta$ and $G$ a graph on $n$ vertices and minimum degree $\left(1-\frac{c}{\Delta}\right) n$. Then any $k$-bounded edge-coloring of $G$ has a rainbow copy of $H$, provided that $k \leq \frac{n}{\beta \Delta^{2}}$.

The theorem can be proved by adding a collection of bad events of size 1 corresponding to the set of non-edges of $G$. Again, as a by-product of the local lemma, the minimum degree condition in Theorem 1 for the existence of a rainbow copy of $H$ is not best possible. We defer the discussion of which is the tight minimum degree condition to the end of the introduction.

In this paper we study the existence of rainbow copies of $H$ in $G$ with asymptotically tight conditions on the minimum degree of $G$, in two particular cases: (1) $H$ is a perfect matching and $G$ is bipartite and (2) $H$ is a Hamiltonian cycle. In both cases the minimum degree threshold is $n / 2$ and it follows from classical results.

Our first result states that, provided there are not many edges with the same color, at the moment the minimum degree ensures the existence of a perfect matching in a bipartite graph, it also ensures the existence of a rainbow one.

Theorem 2. For every $\epsilon>0$, there exist $c>0$ and $n_{0}$ such that for every $n \geq n_{0}$ the following holds: If $G=(A, B)$ is a bipartite graph with $|A|=|B|=n$ and minimum degree at least $(1+\epsilon) \frac{n}{2}$, then every cn-bounded edge-coloring of $G$ contains a rainbow perfect matching.

Theorem 2 has a direct consequence on Latin squares. A Latin square $L$ of size $n$ is an $n \times n$ array with positive integer entries such that each number appears at most once in each row or column. A partial Latin square is a latin square where some positions are blank. A transversal of $L$ is a set of $n$ cells in the array, no two in the same row or column. A transversal is Latin if the integers contained in its cell are pairwise distinct.

Corollary 3. For every $\epsilon>0$, there exist $c>0$ and $n_{0}$ such that for every $n \geq n_{0}$ the following holds: If $L$ is a partial Latin square of size $n$ where each row and column have at least $(1+\epsilon) \frac{n}{2}$ entries and no entry appears more than cn times, then $L$ contains a Latin transversal.

Our second result studies the rainbow Hamiltonian cycles in sparse graphs.

Theorem 4. For every $\epsilon>0$, there exist $c^{\prime}>0$ and $n_{0}$ such that for every $n \geq n_{0}$ the following holds: If $G$ is a graph on $n$ vertices with minimum degree at least $(1+\epsilon) \frac{n}{2}$, then every cn-bounded edge-coloring of $G$ contains a rainbow Hamiltonian cycle.

In contrast to [13, the main ingredient in our proofs is to directly study the probability space given by the set of perfect matchings (or Hamiltonian cycles) of the graph $G$. This allows us to be more precise in the minimum degree condition needed to find the existence of such rainbow subgraph. 
Note that If $n$ is even, Theorem 4 immediately provides the existence of a rainbow matching in the graph. This does not supersede the result of Theorem 2 as the minimum degree condition there is much weaker.

Also note that the condition on the minimum degree in both theorems is asymptotically tight, since there are bipartite graphs with minimum degree $\lceil n / 2\rceil-1$ and no perfect matching, as well as graphs with minimum degree $\lceil n / 2\rceil-1$ and no Hamiltonian cycle. However, the dependence on the number of colors is not best possible. As we already stated, the use of the local lemma forces us to pay a constant factor. The dependence of $c$ in $\epsilon$ is $c=O\left(\epsilon^{2}\right)$ for Theorem 2 and $c=O\left(\epsilon^{3}\right)$ for Theorem 4. We believe that the correct dependence in both cases should be $c=O(\epsilon)$. As a corollary, we obtain that Theorem 2 and 4 are still valid for minimum degree at least $\frac{n}{2}+O(\sqrt{n})$ and $\frac{n}{2}+O\left(n^{2 / 3}\right)$, respectively, provided that the coloring is 2-bounded. We conjecture that any 2-bounded coloring of a bipartite graph with minimum degree at least $\frac{n}{2}$ has a rainbow perfect matching and any 2-bounded coloring of a graph with minimum degree at least $\frac{n}{2}$ has a rainbow Hamiltonian cycle.

An immediate corollary of Theorem 2 follows from the fact that by removing a perfect matching we decrease the minimum degree of the graph by exactly one.

Corollary 5. For every $\epsilon, \gamma>0$, there exist $c>0$ and $n_{0}$ such that for every $n \geq n_{0}$ the following holds: If $G=(A, B)$ is a bipartite graph with $|A|=|B|=n$ and minimum degree at least $\left(\frac{1}{2}+\epsilon\right) n$, then every cn-bounded edge-coloring of $G$ contains $(1-\gamma) \epsilon n$ edge-disjoint rainbow perfect matching.

A similar statement for Hamiltonian cycles also follows from Theorem 4 .

Finally, it would be interesting to study the existence of such rainbow subgraphs under other conditions that ensure the existence of a copy. Two examples are the following:

a) Does every 2-bounded coloring of a regular bipartite graph have a rainbow perfect matching?

b) Does every 2-bounded coloring of a graph satisfying Ore's condition $\left.\right|^{1}$ have a rainbow Hamiltonian cycle?

\subsection{Minimum degree threshold}

A necessary condition for $G$ to contains a rainbow copy of $H$ is to contain at least one copy of $H$. Define $\delta(\Delta, n)$ to be the infimum over all $\delta$ such that every graph $G$ on $n$ vertices and minimum degree at least $\delta n$ contains any graph $H$ on $n$ vertices and maximum degree $\Delta$. Let $\delta(\Delta)=\limsup _{n \rightarrow \infty} \delta(\Delta, n)$.

Bollobás and Eldridge [2] and Catlin [4] independently conjectured that $\delta(\Delta)=1-\frac{1}{\Delta+1}$. The lower bound $\delta(\Delta) \geq 1-\frac{1}{\Delta+1}$ is given by considering $H$ to be the union of $n /(\Delta+1)$ cliques and $G$ a slightly unbalanced $(\Delta+1)$-partite complete graph. Sauer and Spencer [12] proved that $\delta(\Delta) \leq 1-\frac{1}{2 \Delta}$ and this was improved by Kaul and Kostochka [9] to $\delta(\Delta) \leq 1-\frac{3}{5 \Delta}$.

Here we make the following conjecture.

Conjecture 6. For every $\epsilon$ and $\Delta$, there exist $c>0$ and $n_{0}$ such that for every $n \geq n_{0}$ the following holds: If $G$ is a graph on $n$ vertices with minimum degree at least $(1+\epsilon) \delta(\Delta)$ and $H$ is a graph on $n$ vertices with maximum degree at most $\Delta$, then every cn-bounded edge-coloring of $G$ contains a rainbow copy of $H$.

\footnotetext{
${ }^{1}$ A graph $G$ satisfies Ore's condition if for every $x y \notin E(G)$ we have $d(x)+d(y) \geq n$.
} 
While Theorem 2 and 4 are the sparse analogues of the results in [6, 1], Conjecture 6 is the sparse analogue of [3]. We do not dare to conjecture which is the correct dependency of $c$ in terms of $\epsilon$ and $\Delta$.

\section{Notation and Tools}

All graphs are undirected and simple. Given a graph $G$, we denote by $V=V(G)$ it vertex set and by $E=E(G)$ its edge set. We denote by $n$ the size of $V$. For every $x \in V$, we will denote by $N(x)$ the set of vertices $G$ that are adjacent to $x$. We denote by $d(x)$ the size of $N(x)$. Similarly, for a set $S \subseteq V$ we denote by $N(S)$ the union of $N(x)$ for every $x \in S$. We denote by $\delta(G)$ and by $\Delta(G)$ the minimum and maximum degree of the graph $G$, respectively. We use $\log x$ to denote the base 2 logarithm of $x$.

Lemma 7 (Lopsided Lovász Local Lemma [6]). Consider a set $\mathcal{E}$ of (typically bad) events in a probability space $\Omega$. Let $P \in(0,1)$. For every $E \in \mathcal{E}$, consider a set $\mathcal{D}(E)$ of at most $D$ other events in $\mathcal{E}$. Suppose that for all $\mathcal{T} \subseteq \mathcal{E} \backslash(\{E\} \cup \mathcal{D}(E))$ we have that

$$
\mathbb{P}\left[E \mid \bigcap_{F \in \mathcal{T}} F^{c}\right] \leq P .
$$

If $4 P D \leq 1$, then with positive probability, none the events in $\mathcal{E}$ occur.

\section{Proof of Theorem 2}

Our goal is to apply the Lopsided version of the Local Lemma in a similar way as in [6] but to a different probability space. Let $\Omega$ be the space of perfect matchings of $G$ equipped with the uniform measure. Since $\delta(G) \geq n / 2$, it is a direct consequence of Hall's theorem that $\Omega \neq \emptyset$. Let $M \in \Omega$ be a perfect matching of $G$ chosen uniformly at random among all the perfect matchings of $G$.

Let $c=\epsilon^{2} / 32$. Write $k=c n$ and $d=(1+\epsilon) \frac{n}{2}$. Let $\chi: E \rightarrow \mathbb{N}$ be a $k$-bounded edge-coloring of $G$. An unordered pair of edges $a_{1} b_{1}, a_{2} b_{2}$ of $G$ is called dangerous with respect to $\chi$ (or simply, dangerous) if $a_{1} \neq a_{2}, b_{1} \neq b_{2}$ and $\chi\left(a_{1} b_{1}\right)=\chi\left(a_{2} b_{2}\right)$. For every unordered pair $\left\{a_{1} b_{1}, a_{2} b_{2}\right\}$ of dangerous edges, we define the event $E_{a_{1}, a_{2}}^{b_{1}, b_{2}} \subseteq \Omega$ as $a_{1} b_{1}, a_{2} b_{2} \in M$. Note that $E_{a_{1}, a_{2}}^{b_{1}, b_{2}}$ and $E_{a_{2}, a_{1}}^{b_{2}, b_{1}}$ denote the same event. Let $\mathcal{E}$ be the set of events containing all such events for each pair of dangerous edges. Note that if none of the events in $\mathcal{E}$ are satisfied, then no color appears twice in $M$, and $M$ is a rainbow perfect matching of $G$. Our goal will be to prove that the probability none of the events in $\mathcal{E}$ occur is positive, which provides the existence of a rainbow matching.

For every dangerous pair $a_{1} b_{1}, a_{2} b_{2}$, let $\mathcal{D}\left(E_{a_{1}, a_{2}}^{b_{1}, b_{2}}\right)$ be the collection of events of the form $E_{a_{3}, a_{4}}^{b_{3}, b_{4}}$ in $\mathcal{E} \backslash\left\{E_{a_{1}, a_{2}}^{b_{1}, b_{2}}\right\}$, such that the set of edges $\left\{a_{1} b_{1}, a_{2} b_{2}, a_{3} b_{3}, a_{4} b_{4}\right\}$ does not induce a matching in $G$. Let us first bound the size of the sets $\mathcal{D}\left(E_{a_{1}, a_{2}}^{b_{1}, b_{2}}\right)$. Let us upper bound the number of ways to construct $E_{a_{3}, a_{4}}^{b_{3}, b_{4}} \in \mathcal{D}\left(E_{a_{1}, a_{2}}^{b_{1}, b_{2}}\right)$. There are 4 ways to select a vertex in $\left\{a_{1}, a_{2}, b_{1}, b_{2}\right\}$ that intersects $\left\{a_{3}, a_{4}, b_{3}, b_{4}\right\}$. Assume that $a_{3}$ is the chosen one. Then, there are at most $n$ ways to select the edge $a_{3} b_{3}$. Since the coloring is $k$-bounded, there are at most $k-1$ ways to choose an edge $a_{4} b_{4}$ different than $a_{3} b_{3}$ and with color $\chi\left(a_{3} b_{3}\right)$. It follows that

$$
\left|\mathcal{D}\left(E_{a_{1}, a_{2}}^{b_{1}, b_{2}}\right)\right| \leq 4 n(k-1) \leq 4 c n^{2} \leq \frac{\epsilon^{2}}{8} \cdot n^{2}=: D .
$$


The following claim shows that (1) holds for a sufficiently small $P$.

Lemma 8. For every $\mathcal{T} \subseteq \mathcal{E} \backslash\left(\left\{E_{a_{1}, a_{2}}^{b_{1}, b_{2}}\right\} \cup \mathcal{D}\left(E_{a_{1}, a_{2}}^{b_{1}, b_{2}}\right)\right)$, we have

$$
\mathbb{P}\left[E_{a_{1}, a_{2}}^{b_{1}, b_{2}} \mid \bigcap_{F \in \mathcal{T}} F^{c}\right] \leq 2 \epsilon^{-2} / n^{2}=: P .
$$

Assuming that the claim holds, we can conclude. Since for every $E \in \mathcal{E}$ we have $|\mathcal{D}(E)| \leq D=$ $\left(\epsilon^{2} / 8\right) n^{2}$, it follows that $4 P D \leq 1$. So we can use Lemma 7 to conclude that $\mathbb{P}\left[\cap_{E \in \mathcal{E}} E^{c}\right]>0$. Thus, it suffices to prove the claim.

Proof of the claim. We call a matching $M$ of $G$ good if it satisfies $\cap_{F \in \mathcal{T}} F^{c}$. We may assume that there is at least one good matching since otherwise

$$
\mathbb{P}\left[E_{a_{1}, a_{2}}^{b_{1}, b_{2}} \cap \bigcap_{F \in \mathcal{T}} F^{c}\right] \leq \mathbb{P}\left[\bigcap_{F \in \mathcal{T}} F^{c}\right]=0,
$$

and the inequality in (1) holds trivially.

Since $\Omega$ is equipped with the uniform probability measure, we will proceed by a counting argument. Denote by $\mathcal{M}$ the set of all good perfect matchings. Consider the subset $\mathcal{M}_{0} \subseteq \mathcal{M}$ that contains all perfect matchings $M \in \mathcal{M}$ such that $a_{1} b_{1}, a_{2} b_{2} \in M$.

Construct an auxiliary bipartite graph $\mathcal{G}=\left(\mathcal{M}_{0}, \mathcal{M} \backslash \mathcal{M}_{0}\right)$. Two matchings $M_{0} \in \mathcal{M}_{0}$ and $M \in \mathcal{M} \backslash \mathcal{M}_{0}$ are joined by an edge in $\mathcal{G}$ if there exists distinct $a_{3}, a_{4} \in A \backslash\left\{a_{1}, a_{2}\right\}$ and distinct $b_{3}, b_{4} \in B \backslash\left\{b_{1}, b_{2}\right\}$ with $a_{3} b_{3}, a_{4} b_{4} \in M$ such that $a_{1} b_{3}, a_{2} b_{4}, a_{3} b_{1}, a_{4} b_{2} \in M$, and $M_{0}$ and $M$ coincide when restricted to $\left(A \backslash\left\{a_{1}, a_{2}, a_{3}, a_{4}\right\}\right) \times\left(B \backslash\left\{b_{1}, b_{2}, b_{3}, b_{4}\right\}\right)$. In other words, if $M_{0} \cup M$ induces a subgraph composed by a partial matching of size $n-4$ and two vertex-disjoint cycles of length $4: a_{1} b_{1} a_{3} b_{3}$ and $a_{2} b_{2} a_{4} b_{4}$.

Let $\delta\left(\mathcal{M}_{0}\right)$ and $\Delta\left(\mathcal{M} \backslash \mathcal{M}_{0}\right)$ be the minimum degree of $\mathcal{M}_{0}$ and the maximum degree of $\mathcal{M} \backslash \mathcal{M}_{0}$, respectively. By double-counting the number of edges of $\mathcal{G}$ we obtain

$$
\delta\left(\mathcal{M}_{0}\right)\left|\mathcal{M}_{0}\right| \leq e(\mathcal{G}) \leq \Delta\left(\mathcal{M} \backslash \mathcal{M}_{0}\right)\left|\mathcal{M} \backslash \mathcal{M}_{0}\right|
$$

It follows that,

$$
\mathbb{P}\left[E_{a_{1}, a_{2}}^{b_{1}, b_{2}} \mid \bigcap_{F \in \mathcal{T}} F^{c}\right]=\frac{\left|\mathcal{M}_{0}\right|}{\left|\mathcal{M}_{0}\right|+\left|\mathcal{M} \backslash \mathcal{M}_{0}\right|} \leq \frac{\left|\mathcal{M}_{0}\right|}{\left|\mathcal{M} \backslash \mathcal{M}_{0}\right|} \leq \frac{\Delta\left(\mathcal{M} \backslash \mathcal{M}_{0}\right)}{\delta\left(\mathcal{M}_{0}\right)}
$$

Let us bound the two degrees. We first lower bound $\delta\left(\mathcal{M}_{0}\right)$. Recall that, given $M_{0} \in \mathcal{M}_{0}$ with $a_{1} b_{1}, a_{2} b_{2} \in M_{0}$ and two cycles of length 4 of the form $a_{1} b_{1} a_{3} b_{3}$ and $a_{2} b_{2} a_{4} b_{4}$ with $a_{3} b_{3}, a_{4} b_{4} \in M_{0}$, there exists a matching $M$ such that $M_{0}$ and $M$ are adjacent in $\mathcal{G}$. It remains to lower bound the number of ways to find such cycles. For $S \subseteq A$, let $M_{0}(S) \subseteq B$ be the vertices of $B$ that are matched to $S$ by $M_{0}$. For every $b_{3} \in\left(N\left(a_{1}\right) \cap M_{0}\left(N\left(b_{1}\right)\right)\right) \backslash\left\{b_{1}, b_{2}\right\}$ with $a_{3}=M_{0}^{-1}\left(b_{3}\right) \in N\left(b_{1}\right)$, we obtain a cycle $a_{1} b_{1} a_{3} b_{3}$ that satisfies our conditions. Moreover, since the minimum degree of $G$ is at least $(1+\epsilon) \frac{n}{2}$,

$$
\begin{aligned}
\left|\left(N\left(a_{1}\right) \cap M_{0}\left(N\left(b_{1}\right)\right)\right) \backslash\left\{b_{1}, b_{2}\right\}\right| & =\left|N\left(a_{1}\right)\right|+\left|M_{0}\left(N\left(b_{1}\right)\right)\right|-\left|N\left(a_{1}\right) \cup M_{0}\left(N\left(b_{1}\right)\right)\right|-2 \\
& \geq\left|N\left(a_{1}\right)\right|+\left|N\left(b_{1}\right)\right|-n-2 \\
& \geq \epsilon n-2 .
\end{aligned}
$$


Thus, $a_{1} b_{1}$ is contained in at least $\epsilon n-2$ cycles of length 4 that contain another edge from $M_{0}$. An analogous argument shows that, given that the cycle $a_{1} b_{1} a_{3} b_{3}$ has been chosen, $a_{2} b_{2}$ is contained in at least $\epsilon n-3$ cycles of length 4 disjoint from the previous one. We conclude that $\delta\left(\mathcal{M}_{0}\right) \geq$ $(\epsilon n-2)(\epsilon n-3) \geq \epsilon^{2} n^{2} / 2$.

Now we proceed to upper bound $\Delta\left(\mathcal{M} \backslash \mathcal{M}_{0}\right)$. Given a matching $M \in \mathcal{M} \backslash \mathcal{M}_{0}$ there is at most one way to select two cycles of length 4 such that $a_{1}$ and $b_{1}$ belong to the first cycle and $a_{2}$ and $b_{2}$ belong to the second one. Thus, $\Delta\left(\mathcal{M} \backslash \mathcal{M}_{0}\right) \leq 1$.

We conclude that

$$
P:=\mathbb{P}\left[E_{a_{1}, a_{2}}^{b_{1}, b_{2}} \mid \bigcap_{F \in \mathcal{T}} F^{c}\right] \leq \frac{2 \epsilon^{-2}}{n^{2}} .
$$

\section{Proof of Theorem 4}

Let $\Omega$ be the space of Hamiltonian cycles of $G$ equipped with the uniform measure. Since $\delta(G) \geq$ $(1+\epsilon) \frac{n}{2} \geq \frac{n}{2}$, by Dirac's theorem $\Omega \neq \emptyset$. Let $H \in \Omega$ be a Hamiltonian cycle chosen uniformly at random among all Hamiltonian cycles of $G$.

Let $c=\left(\epsilon^{3} / 8000\right) \cdot n$ and write $k=c n$. Let $\chi: E \rightarrow \mathbb{N}$ be a $k$-bounded edge-coloring of $G$. We slightly modify the definition of dangerous pair of edges. An unordered pair of edges $x_{1} y_{1}, x_{2} y_{2}$ of $G$ is called dangerous if $\chi\left(x_{1} y_{1}\right)=\chi\left(x_{2} y_{2}\right)$. The main difference with respect to the previous section, is that here we allow $x_{1}=y_{2}$.

For every dangerous pair $x_{1} y_{1}, x_{2} y_{2}$ of dangerous edges, we define the event $E_{x_{1}, x_{2}}^{y_{1}, y_{2}} \subseteq \Omega$ as $x_{1} y_{1}, x_{2} y_{2} \in H$. Let $\mathcal{E}$ be the collection of events defined by pairs of dangerous edges. Again, in order to provide the existence of a rainbow Hamiltonian cycle, we aim to prove that with positive probability, none of the events in $\mathcal{E}$ hold. We also consider $\mathcal{D}\left(E_{x_{1}, x_{2}}^{y_{1}, y_{2}}\right)$, the set of events of the form $E_{u_{1}, u_{2}}^{v_{1}, v_{2}}$ in $\mathcal{E} \backslash\left\{E_{x_{1}, x_{2}}^{y_{1}, y_{2}}\right\}$ such that $\left\{x_{1}, y_{1}, x_{2}, y_{2}\right\} \cap\left\{u_{1}, v_{1}, u_{2}, v_{2}\right\} \neq \emptyset$.

Recall that the coloring is $k$-bounded. Given $x_{1}, x_{2}, y_{1}, y_{2}$, the number of ways we can select a dangerous pair of edges $u_{1} v_{1}, u_{2} v_{2}$ such that one of them is incident to at least one vertex in $\left\{x_{1}, x_{2}, y_{1}, y_{2}\right\}$ is

$$
\left|\mathcal{D}\left(E_{x_{1}, x_{2}}^{y_{1}, y_{2}}\right)\right| \leq 4 n(k-1) \leq 4 c n^{2} \leq \frac{\epsilon^{3}}{8000} \cdot n^{2}=: D .
$$

Before bounding the conditional probability of an event, we present the following technical lemma.

Lemma 9. Let $A, B \subseteq[n]$ such that $|A|+|B| \geq(1+\gamma) n$. Then there exist at least $\gamma^{2} n / 20$ pairs $\left(a_{1}, a_{2}\right) \in A \times A$ with $\left|a_{1}-a_{2}\right| \leq \gamma^{-1}$ such that $a_{1}+1 \in B$ and $a_{2}-1 \in B$.

Proof. Write $\ell=\left\lceil\gamma^{-1} / 4\right\rceil$. Let $\mathcal{S}=\left\{S_{1}, \ldots, S_{t}, S_{t+1}\right\}$ be a partition of $[n]$ into $t=\lfloor n / \ell\rfloor$ consecutive intervals of size $\ell$ and a (possibly empty) interval $S_{t+1}$ of size at most $\ell$. In other words, $S_{i}=$ $[(i-1) \ell+1, i \ell]$ for every $1 \leq i \leq t$ and $S_{t+1}=[t \ell+1, n]$. We claim that there exists a set of indices $\mathcal{I} \subseteq[t]$ such that $|\mathcal{I}| \geq \gamma t / 3$ and for every $i \in \mathcal{I}$ we have

$$
\left|A \cap S_{i}\right|+\left|B \cap S_{i}\right| \geq \ell+2 .
$$


Suppose this is not the case. Then

$$
\begin{aligned}
|A|+|B| & =\sum_{i=1}^{t}\left|A \cap S_{i}\right|+\left|B \cap S_{i}\right| \\
& \leq \frac{\gamma}{3} t \cdot 2 \ell+\left(1-\frac{\gamma}{3}\right) t(\ell+2) \\
& \leq t \ell+\left(\frac{\gamma}{3} \ell+2\right) t \\
& \leq n+\left(\frac{\gamma}{3}+\frac{2}{\ell}\right) n \\
& <(1+\gamma) n
\end{aligned}
$$

contradicting the hypothesis of the lemma.

Fix $i \in \mathcal{I}$ and denote by $A_{i}=A \cap S_{i}$ and by $B_{i}=B \cap S_{i}$. We are going to show that there exist $a_{1}, a_{2} \in A_{i}$ such that $a_{1}-1$ and $a_{2}+1$ belong to $B_{i}$. We do not insist that $a_{1} \neq a_{2}$.

Let $A_{i}+1=\left\{a+1: a \in A_{i}\right\} \cap S_{i}$ and note that $\left|A_{i}+1\right| \geq\left|A_{i}\right|-1$. Recall that $i \in \mathcal{I}$ implies $\left|A_{i}\right|+\left|B_{i}\right| \geq \ell+2$. Since $\left|\left(A_{i}+1\right) \cup B_{i}\right| \leq\left|S_{i}\right| \leq \ell$ and using (5), we have

$$
\begin{aligned}
\left|\left(A_{i}+1\right) \cap B_{i}\right| & =\left|A_{i}+1\right|+\left|B_{i}\right|-\left|\left(A_{i}+1\right) \cup B_{i}\right| \\
& \geq\left|A_{i}\right|-1+\left|B_{i}\right|-\left|\left(A_{i}+1\right) \cup B_{i}\right| \\
& \geq 1 .
\end{aligned}
$$

Since this intersection is non-empty, we let $b_{1} \in B_{i}$ be an element of it, and $a_{1}=b_{1}-1 \in A_{i}$. Analogously, we can find $a_{2} \in A_{i}$ and $b_{2} \in B_{i}$ with $a_{2}=b_{2}+1$.

Observe that $\left|a_{1}-a_{2}\right| \leq\left|S_{i}\right|=\ell \leq \gamma^{-1}$, as desired. Moreover, since the sets $S_{i}$ are pairwise disjoint, we can find at least $|\mathcal{I}| \geq \gamma t / 3=\gamma^{2} n / 20$ of such pairs $\left(a_{1}, a_{2}\right)$, concluding the proof of the lemma.

We can now state the corresponding bound on the conditional probability claim shows that (1) holds for a sufficiently small $P$.

Lemma 10. For every $\mathcal{T} \subseteq \mathcal{E} \backslash\left(\left\{E_{x_{1}, x_{2}}^{y_{1}, y_{2}}\right\} \cup \mathcal{D}\left(E_{x_{1}, x_{2}}^{y_{1}, y_{2}}\right)\right)$, we have

$$
\mathbb{P}\left[E_{x_{1}, x_{2}}^{y_{1}, y_{2}} \mid \bigcap_{F \in \mathcal{T}} F^{c}\right] \leq \frac{2000 \epsilon^{-3}}{n^{2}}:=P .
$$

Assuming that the claim holds, we obtain $4 P D \leq 1$ and we use Lemma 7 to conclude that $\mathbb{P}\left[\cap_{E \in \mathcal{E}} E^{c}\right]>0$.

Proof of the claim. Let $H$ be a Hamiltonian cycle of $G$ that contains the edges $x_{1} y_{1}$ and $x_{2} y_{2}$. Recall that we allow for $x_{1}=y_{2}$. We introduce an operation on the edges of $H$ that will give rise to another 2-regular subgraph of $G$. This subgraph is not always a Hamiltonian cycle.

First, orient the edges of $H$ in an arbitrary and cyclic order. We denote by $\vec{H}$ the oriented Hamiltonian cycle. We may assume that the edges $x_{1} y_{1}$ and $x_{2} y_{2}$ are traversed from $x_{i}$ to $y_{i}$ in $\vec{H}$. Otherwise, we can reverse the cyclic order of $\vec{H}$. Let $H_{x_{1}, x_{2}}^{y_{1}, y_{2}}$ be the Hamiltonian cycle $H$ where the ordered pair of edges $\left(x_{1} y_{1}, x_{2} y_{2}\right)$ is labelled.

We now define two different type of operations, called switchings and denoted by $s($.$) and t($. respectively, that transform a Hamiltonian cycle $H_{x_{1}, x_{2}}^{y_{1}, y_{2}}$ into a 2-regular spanning subgraph of $G$. 
- Switching of type $s$ : for every edge $u_{1} v_{1} \in E(\vec{H})$ such that $u_{1} \in N\left(x_{2}\right)$ and $v_{1} \in N\left(y_{2}\right)$, and every edge $u_{2} v_{2} \in E(\vec{H})$ such that $u_{2} \in N\left(x_{1}\right)$ and $v_{2} \in N\left(y_{1}\right)$, we construct $s_{u_{1}, u_{2}}^{v_{1}, v_{2}}\left(H_{x_{1}, x_{2}}^{y_{1}, y_{2}}\right)$ as follows: delete the edges $x_{1} y_{1}, x_{2} y_{2}, u_{1} v_{1}$ and $u_{2} v_{2}$ from $H$ and add the edges $x_{1} u_{2}, x_{2} u_{1}, y_{1} v_{2}$ and $y_{2} v_{1}$.

Note that the resulting spanning subgraph $s_{u_{1}, u_{2}}^{v_{1}, v_{2}}\left(H_{x_{1}, x_{2}}^{y_{1}, y_{2}}\right)$ is a 2-regular multigraph (loops and double edges may appear if some endpoints of the edges coincide). Moreover, $s_{u_{1}, u_{2}}^{v_{1}, v_{2}}\left(H_{x_{1}, x_{2}}^{y_{1}, y_{2}}\right)$ is a Hamiltonian cycle if $H$ is of the form,

$$
x_{1}, y_{1}, \ldots, u_{1}, v_{1}, \ldots, x_{2}, y_{2}, \ldots, u_{2}, v_{2}, \ldots, x_{1}
$$

or of the form,

$$
x_{1}, y_{1}, \ldots, u_{2}, v_{2}, \ldots, u_{1}, v_{1}, \ldots, x_{2}, y_{2}, \ldots, x_{1}
$$

- Switching of type $t$ : for every edge $u_{1} v_{1} \in E(\vec{H})$ such that $u_{1} \in N\left(x_{2}\right)$ and $v_{1} \in N\left(y_{2}\right)$, and every edge $v_{2} u_{2} \in E(\vec{H})$ such that $u_{2} \in N\left(x_{1}\right)$ and $v_{2} \in N\left(y_{1}\right)$, we construct $t_{u_{1}, u_{2}}^{v_{1}, v_{2}}\left(H_{x_{1}, x_{2}}^{y_{1}, y_{2}}\right)$ as follows: delete the edges $x_{1} y_{1}, x_{2} y_{2}, u_{1} v_{1}$ and $u_{2} v_{2}$ from $H$ and add the edges $x_{1} u_{2}, x_{2} u_{1}, y_{1} v_{2}$ and $y_{2} v_{1}$.

As before, the resulting spanning subgraph $t_{u_{1}, u_{2}}^{v_{1}, v_{2}}\left(H_{x_{1}, x_{2}}^{y_{1}, y_{2}}\right)$ is a 2-regular multigraph and it is Hamiltonian cycle if $H$ is of the form

$$
x_{1}, y_{1}, \ldots, u_{1}, v_{1}, \ldots, v_{2}, u_{2}, \ldots, x_{2}, y_{2}, \ldots, x_{1}
$$
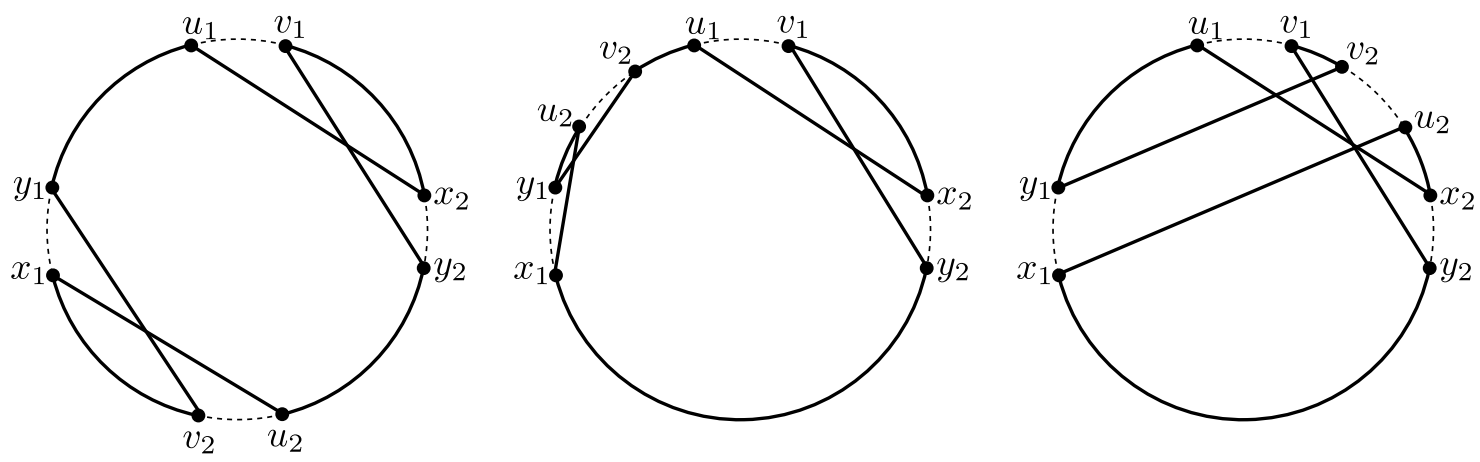

Figure 1: Cases (6), (7) and (8).

The following claim shows that there are many switchings that transform $H_{x_{1}, x_{2}}^{y_{1}, y_{2}}$ into a Hamiltonian cycle.

Claim A. For every edge $u_{1} v_{1} \in E(\vec{H})$, there exist at least $\epsilon^{2} n / 30$ edges $u_{2} \in E(\vec{H})$ such that either $s_{u_{1}, u_{2}}^{v_{1}, v_{2}}\left(H_{x_{1}, x_{2}}^{y_{1}, y_{2}}\right)$ or $t_{u_{1}, u_{2}}^{v_{1}, v_{2}}\left(H_{x_{1}, x_{2}}^{y_{1}, y_{2}}\right)$ is a Hamiltonian cycle.

Proof. Without loss of generality, we can assume $\vec{H}$ follows the cyclic order

$$
x_{1}, y_{1}, \ldots, u_{1}, v_{1}, \ldots, x_{2}, y_{2}, \ldots, x_{1}
$$

otherwise we can obtain it by relabelling the edges $x_{1} y_{1}$ and $x_{2} y_{2}$. 
Select an arbitrary vertex $u \in V$, and let $\left(u=h_{1}, \ldots, h_{n}, h_{1}\right)$ be the vertices of the Hamiltonian cycle ordered following the chosen orientation and starting at $u$. Let $A:=\left\{i \in[n]: h_{i} \in N\left(x_{1}\right)\right\}$ and $B:=\left\{i \in[n]: h_{i} \in N\left(y_{1}\right)\right\}$. By the hypothesis of the theorem, we have $|A|+|B| \geq(1+\epsilon) n$. By Lemma 9 with $\gamma=\epsilon$, there exist a set $\mathcal{S}$ of pairs $\left(a_{1}, a_{2}\right) \in A \times A$ with $\left|a_{1}-a_{2}\right| \leq \epsilon^{-1}$ such that $a_{1}+1 \in B$ and $a_{2}-1 \in B$, with $|\mathcal{S}| \geq \frac{\epsilon^{2} n}{20}$.

For each $\left(a_{1}, a_{2}\right) \in \mathcal{S}$, if $w_{2}=h_{a_{1}}, z_{2}=h_{a_{1}+1}$, then $s_{u_{1}, w_{2}}^{v_{1}, z_{2}}\left(H_{x_{1}, x_{2}}^{y_{1}, y_{2}}\right)$ is well-defined. Similarly, if $w_{2}^{\prime}=h_{a_{2}}, z_{2}^{\prime}=h_{a_{2}-1}$, then $t_{u_{1}, w_{2}^{\prime}}^{v_{1}, z^{\prime}}\left(H_{x_{1}, x_{2}}^{y_{1}, y_{2}}\right)$ is also well-defined.

Now partition $H \backslash\left\{x_{1} y_{1}, u_{1} v_{1}, x_{2} y_{2}\right\}$ into three paths: $P_{1}=y_{1}, \ldots, u_{1}, P_{2}=v_{1}, \ldots, x_{2}$ and $P_{3}=y_{2}, \ldots, x_{1}$; and note that some of these paths might be edgeless. We say that a pair $\left(c_{1}, c_{2}\right) \in$ $[n] \times[n]$ is valid if both $h_{c_{1}} h_{c_{1}+1}$ and $h_{c_{2}-1} h_{c_{2}}$ belong to the same path.

Recall that if $\left(a_{1}, a_{2}\right) \in \mathcal{S}$, then $\left|a_{1}-a_{2}\right| \leq \epsilon^{-1}$. Thus, there are at most $3 \epsilon^{-2}$ pairs in $\mathcal{S}$ that are not valid. Thus, the number of valid pairs in $\mathcal{S}$ is at least

$$
\frac{\epsilon^{2} n^{2}}{20}-3 \epsilon^{-2} \geq \frac{\epsilon^{2} n^{2}}{30},
$$

Fix a valid pair $\left(a_{1}, a_{2}\right) \in \mathcal{S}$. If $h_{a_{1}} h_{a_{1}+1}$ and $h_{a_{2}-1} h_{a_{2}}$ are either in $P_{1}$ or in $P_{3}$, then, by (6) or by (7), $s_{u_{1}, h_{a_{1}}}^{v_{1}, h_{a_{1}+1}}\left(H_{x_{1}, x_{2}}^{y_{1}, y_{2}}\right)$ is a Hamiltonian cycle. If these edges are in $P_{2}$, then, by $(8), t_{u_{1}, h_{a_{2}}}^{v_{1}, h_{a_{2}}}\left(H_{x_{1}, x_{2}}^{y_{1}, y_{2}}\right)$, is also a Hamiltonian cycle.

We conclude that there are at least $\frac{\epsilon^{2} n^{2}}{30}$ choices for $u_{2} v_{2}$ such that either $s_{u_{1}, u_{2}}^{v_{1}, v_{2}}\left(H_{x_{1}, x_{2}}^{y_{1}, y_{2}}\right)$ or $t_{u_{1}, u_{2}}^{v_{1}, v_{2}}\left(H_{x_{1}, x_{2}}^{y_{1}, y_{2}}\right)$ is a Hamiltonian cycle.

Recall that, given $x_{1} y_{1}, x_{2} y_{2}$ and a set $\mathcal{T} \subseteq \mathcal{E} \backslash\left(\left\{E_{x_{1}, x_{2}}^{y_{1}, y_{2}}\right\} \cup \mathcal{D}\left(E_{x_{1}, x_{2}}^{y_{1}, y_{2}}\right)\right)$, we say that a Hamiltonian cycle is good if it satisfies $\cap_{F \in \mathcal{T}} F^{c}$.

We now construct an auxiliary bipartite graph $\mathcal{H}=(\mathcal{A}, \mathcal{B})$, where $\mathcal{A}$ is composed by all the good Hamiltonian cycles $G$ that contain both $x_{1} y_{1}$ and $x_{2} y_{2}$, and where $\mathcal{B}$ is composed by all the good Hamiltonian cycles of $G$ that contain neither $x_{1} y_{1}$ nor $x_{2} y_{2}$. We add edges between $H \in \mathcal{A}$ and $H^{\prime} \in \mathcal{B}$ if there exists a switching that transforms $H$ into $H^{\prime}$, and vice versa.

By double-counting of the edges of $\mathcal{H}$ in a similar way as in the proof of Theorem 2, it follows that

$$
\mathbb{P}\left[E_{x_{1}, x_{2}}^{y_{1}, y_{2}} \mid \cap_{F \in \mathcal{T}} F^{c}\right]=\frac{|\mathcal{A}|}{|\mathcal{A}|+|\mathcal{B}|} \leq \frac{|\mathcal{A}|}{|\mathcal{B}|} \leq \frac{\Delta(\mathcal{B})}{\delta(\mathcal{A})}
$$

where $\delta(\mathcal{A})$ and $\Delta(\mathcal{B})$ are the minimum degree in $\mathcal{A}$ and the maximum degree in $\mathcal{B}$, respectively.

Let $H^{\prime} \in \mathcal{B}$. To count the number of cycles in $\mathcal{A}$ that can give rise to $\mathcal{H}$, we need to select 4 edges in $E\left(H^{\prime}\right)$ adjacent to $x_{1}, y_{1}, x_{2}$ and $y_{2}$, respectively; this can be done in $2^{4}$ ways since the degree of each vertex in $H^{\prime}$ is 2 . Once we have decided if $H^{\prime}$ was created from a Hamiltonian cycle in $\mathcal{A}$ using a switching of type $s$ or of type $t$, the graph $H$ is determined. Thus, we have $\Delta(\mathcal{B}) \leq 2 \times 2^{4}=32$.

Let $H \in \mathcal{A}$ and let $h_{1}, \ldots h_{n}$ be a cyclic ordering of $V$ according to $H$. Let $A:=\{i \in[n]$ : $\left.h_{i} \in N\left(x_{2}\right)\right\}$ and let $B:=\left\{i \in[n]: h_{i+1} \in N\left(y_{2}\right)\right\}$. Since $|A|,|B| \geq(1+\epsilon) \frac{n}{2}$, there are at least $|A \cap B|-2 \geq \epsilon n-2 \geq \epsilon n / 2$ ways to select $u_{1} v_{1}$ with $u_{1} \in N\left(x_{2}\right), v_{1} \in N\left(y_{2}\right)$ and different from $x_{1} y_{1}$ and $x_{2} y_{2}$. Once $u_{1} v_{1}$ has been fixed, by Claim A, there are at least $\epsilon^{2} n / 30$ choices for an edge $u_{2} v_{2}$ such that either $s_{u_{1}, u_{2}}^{v_{1}, v_{2}}\left(H_{x_{1}, x_{2}}^{y_{1}, y_{2}}\right)$ or $t_{u_{1}, u_{2}}^{v_{1}, v_{2}}\left(H_{x_{1}, x_{2}}^{y_{1}, y_{2}}\right)$ gives rise to a Hamiltonian cycle $H^{\prime}$. By construction, $H^{\prime}$ does not contain the edges $x_{1} y_{1}$ and $x_{2} y_{2}$; that is, $H^{\prime} \in \mathcal{B}$. Thus, we have $\delta(\mathcal{A}) \geq \epsilon^{3} n^{2} / 60$ : 
Using (9) we conclude

$$
\mathbb{P}\left[E_{x_{1}, x_{2}}^{y_{1}, y_{2}} \mid \bigcap_{F \in \mathcal{T}} F^{c}\right] \leq \frac{60 \cdot 32 \epsilon^{-3}}{n^{2}} \leq \frac{2000 \epsilon^{-3}}{n^{2}}
$$

\section{References}

[1] Michael Albert, Alan Frieze, and Bruce Reed, Multicoloured hamilton cycles, Electronic Journal of Combinatorics 2 (1995), no. R10, 1-13.

[2] Béla Bollobás and Stephen E Eldridge, Packings of graphs and applications to computational complexity, Journal of Combinatorial Theory, Series B 25 (1978), no. 2, 105-124.

[3] Julia Böttcher, Yoshiharu Kohayakawa, and Aldo Procacci, Properly coloured copies and rainbow copies of large graphs with small maximum degree, Random Structures \& Algorithms 40 (2012), no. 4, 425-436.

[4] Paul Allen Catlin, Embedding subgraphs and coloring graphs under extremal degree conditions, 1976.

[5] Andrzej Dudek, Alan Frieze, and Andrzej Rucinski, Rainbow hamilton cycles in uniform hypergraphs, Electronic Journal of Combinatorics 19 (2011), no. 1, P46.

[6] Paul Erdős and Joel Spencer, Lopsided lovász local lemma and latin transversals, Discrete Applied Mathematics 30 (1991), no. 2, 151-154.

[7] Alan Frieze and Michael Krivelevich, On rainbow trees and cycles, Electron. J. Combin 15 (2008), no. $1,9$.

[8] Nina Kamčev, Benny Sudakov, and Jan Volec, Bounded colorings of multipartite graphs and hypergraphs, arXiv preprint arXiv:1601.02271 (2016).

[9] Hemanshu Kaul and Alexandr Kostochka, Extremal graphs for a graph packing theorem of sauer and spencer, Combinatorics, Probability and Computing 16 (2007), no. 03, 409-416.

[10] Linyuan Lu and László Székely, Using lovász local lemma in the space of random injections, Electron. J. Combin 14 (2007), no. 1, 13.

[11] H.J. Ryser, Neuere probleme der kombinatorik, (1967).

[12] Norbert Sauer and Joel Spencer, Edge disjoint placement of graphs, Journal of Combinatorial Theory, Series B 25 (1978), no. 3, 295-302.

[13] Benny Sudakov and Jan Volec, Properly colored and rainbow copies of graphs with few cherries, arXiv preprint arXiv:1504.06176 (2015). 